\title{
P2P Based Architecture for Global Home Agent Dynamic Discovery in IP Mobility
}

\author{
Rubén Cuevas, Carmen Guerrero, Ángel Cuevas, María Calderón, Carlos J. Bernardos \\ Departamento de Ingeniería Telemática, Universidad Carlos III de Madrid, Spain \\ Email: $\{$ rcuevas, guerrero, acrumin, maria, cjbc $\} @$ it.uc3m.es
}

\begin{abstract}
Mobility in packet networks has become a critical issue in the last years. Mobile IP and the Network Mobility Basic Support Protocol are the IETF proposals to provide mobility. However, both of them introduce performance limitations, due to the presence of an entity (Home Agent) in the communication path. Those problems have been tried to be solved in different ways. A family of solutions has been proposed in order to mitigate those problems by allowing mobile devices to use several geographically distributed Home Agents (thus making shorter the communication path). These techniques require a method to discover a close Home Agent, among those geographically distributed, to the mobile device. This paper proposes a peer-topeer based solution, called Peer-to-Peer Home Agent Network, in order to discover a close Home Agent. The proposed solution is simple, fully global, dynamic and it can be developed in IPv4 and IPv6.
\end{abstract}

\section{INTRODUCTION}

Cellular Networks and Satellite Networks are examples of different networks where mobility is required to support voice services. Besides, an increasing interest for data and voice integration is happening during the last years, either in mobile or fixed environments. The result of both facts was to adopt the use of packet networks to support at the same time voice and data. The most accepted solution is to utilize IP technology (since almost every network around the world is IP based) as packet support for voice and data integration. Therefore, mobility in IP networks has recently become a necessity in order to support voice communications.

This paper focuses on Mobile IP solutions. Mobile IP [1] [2] is the mechanism proposed by the Internet Engineering Task Force ${ }^{1}$ (IETF) to enable host mobility. However, mobility is also required for vehicles (planes, trains, etc.).Vehicles are considered networks since they have several terminals with some kind of infrastructure inside. Hence, support for network mobility is required. The Network Mobility (NEMO) Basic Support Protocol [3] [4] is the IETF proposal to provide network mobility support.

The basic solution of Mobile IP and NEMO presents the socalled triangular-routing as the main performance limitation: mobile nodes' communications must pass through an entity, called the Home Agent (HA). It is possible that some communications suffer from higher delays than those required by some kind of applications (e.g. real time applications like voice or video) in order to obtain an acceptable performance.

\footnotetext{
${ }^{1}$ http: //www.ietf.org/
}

Several solutions have been proposed in order to solve the routing problems of basic NEMO and Mobile IP. One family of solutions proposes (so as to improve the routing) to reduce the distance between the HA and the mobile devices as much as possible, minimizing the total path length. Basically, in these proposals, a mobile device always tries to use a close HA which permits that mobile device to fulfil the requirements of its communications.

Therefore, this family of solutions needs a mechanism to efficiently discover a close HA. This paper presents a mechanism which solves that problem.

The paper proposes the use of an overlay Peer-to-Peer network (Peer-to-Peer HA Network), formed by HAs, in order to discover a close HA to a certain mobile device. In a nutshell, a mobile node detects that its current HA is too far. Then, it asks its current HA, which belongs to the Peer-to-Peer HA network (P2PHAN), for a close HA. The P2PHAN, based on Border Gateway Protocol (BGP) [5] information, discovers HAs located in the same Autonomous System (AS) as the mobile device. This information is received by the current HA. After that, it sends the list of HAs to the mobile node which finally associates with one of the HA in the list. The proposed solution is simple, fully global, dynamic and it can be deployed in IPv4 and IPv6.

The paper is structured as follows. Section II introduces a brief summary about the technologies involved in this paper, Peer-to-Peer and NEMO. In section III, the proposed solution - called Peer-to-Peer Home Agent Network - is described in detail. Section IV discusses about the related work and Section $\mathrm{V}$ provides some conclusions and introduces further work.

\section{BACKGROUND}

\section{II-A. Network Mobility}

The IETF standardized a solution, called NEMO Basic Support Protocol (specified in the RFC 3963 [3]), to provide network mobility support. This solution defines a mobile network (known also as Network that Moves - $\mathrm{NEMO}^{2}$ ) as a network whose attachment point to the Internet varies with time. The router within the NEMO that connects to the Internet is called the Mobile Router (MR). It is assumed that the NEMO has a Home Network where it resides when it is not moving. Since the NEMO is part of the Home Network, the

\footnotetext{
${ }^{2}$ NEMO can mean NEtwork MObility or NEtwork that MOves according to the context.
} 


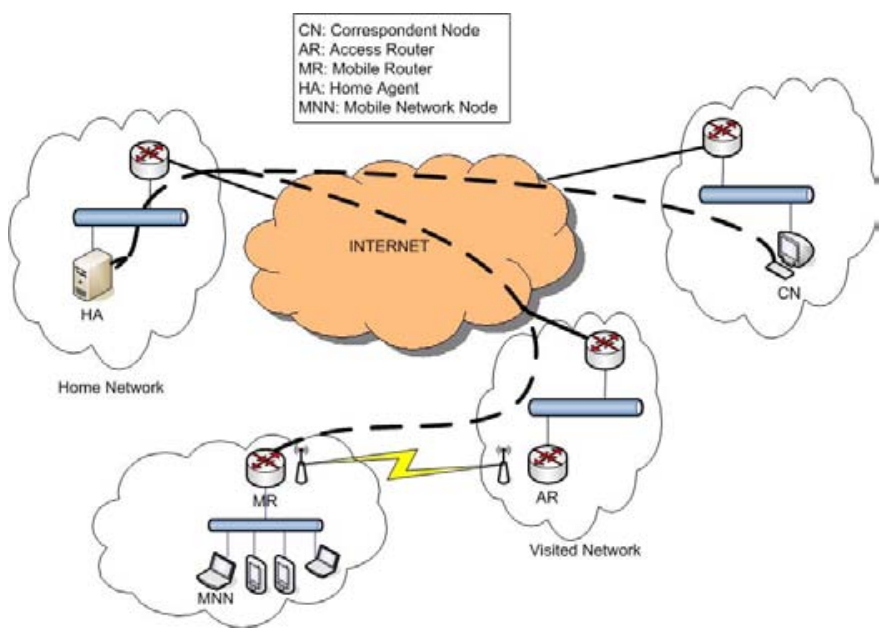

Fig. 1. Basic NEMO Basic Support Protocol operation

Mobile Network has configured addresses belonging to one or more address blocks assigned to the Home Network: the Mobile Network Prefixes (MNPs). These addresses remain assigned to the NEMO when it is away from home. Of course, these addresses only have topological meaning when the NEMO is at home. When the NEMO is away from home, packets addressed to the Mobile Network Nodes (MNNs) will still be routed to the Home Network. Additionally, when the NEMO is away from home, i.e. it is in a visited network, the MR acquires an address from the visited network, called the Care-of Address (CoA), where the routing architecture can deliver packets without additional mechanisms.

The goal of the network mobility support mechanisms is to preserve established communications between the MNNs and external Correspondent Nodes (CNs) despite movement. Packets of such communications will be addressed to the MNNs addresses, which belong to the MNP, so additional mechanisms to forward packets between the Home Network and the NEMO are needed. The basic solution for network mobility support essentially creates a bi-directional tunnel between a special node located in the Home Network of the NEMO (the Home Agent), and the Care-of Address of the MR. Fig. 1 shows the basic operation of the NEMO Basic Support Protocol.

Inefficient routing due to the triangular $\mathrm{CN}-\mathrm{HA}-\mathrm{MR}$ path could introduce delay not desirable in some kind of applications (e.g. real time applications). Therefore, several mechanisms (the so-called Route Optimization - RO - for NEMO solutions) have been proposed in order to improve the sub-optimal routing introduced by the NEMO Basic Support Protocol.

A family of solutions [8] proposes that after the communication set up (which must be done through the HA) the rest of the communication takes place directly between the MR and the $\mathrm{CN}$. The MR informs the $\mathrm{CN}$ about its CoA changes while moving so that the session can be maintained.

Another family of solutions, e.g. Global Home Agent to Home Agent [6] or Scalable Support for Globally Moving
Networks [7], proposes to make the path between HA and MR as shorter as possible. Basically, in these solutions, the MR uses a close HA instead of using always the HA located at its Home Network. By doing so, the triangular path length is reduced. This family of solutions needs a mechanism to discover a closer HA. This paper introduces a mechanism which solves that problem in a global and dynamic manner.

\section{II-B. Peer to peer network}

A Peer-to-Peer (p2p) network [9] is composed by nodes which are both client and server at the same time. These nodes are called peers.

A p2p network permits to distribute information among peers resulting in a distributed storage system. The main features of $\mathrm{p} 2 \mathrm{p}$ networks are their robustness and fault tolerance. Besides, a huge amount of data can be stored, and it offers a quick search of the desired information.

Initially, $p 2 p$ networks were designed to provide file sharing, but many research groups are working to apply p2p in different areas because it is a very versatile technology. For instance, this paper applies $p 2 p$ technology for global HA dynamic discovery. Different types of $p 2 p$ networks have been defined based on several criteria. A complete classification can be found in [9].

Most research efforts have focused on self-organized, fully decentralized, with semantic free indexes $\mathrm{p} 2 \mathrm{p}$ networks, also called Distributed Hash Table p2p networks (DHT-p2p networks). The main features of this kind of $\mathrm{p} 2 \mathrm{p}$ networks are: peers structure the network topology by themselves, the use of a decentralized search method and the use of indexes or keys ${ }^{3}$ (that usually are a set of bits) with no semantic meaning. They can be organized following different structures (ring, tree, ...).

DHT-p2p networks work as follows. There is a registration phase when a node joins the network. It chooses a peer identifier (Peer-ID) which is a set of bits with a length equal to the search keys length.

After that, the peer publishes its objects within the $p 2 p$ network. It hashes the object in order to obtain its key, $k e y=h a s h($ object $)$. Then, it sends the pair $<$ key, its IP address $>$ to the peer with the most similar Peer-ID to that key which stores the pair. Hence, each peer within the p2p network stores pairs $<$ key, peer-owner $I P>$.

The search method is as follows. Peer $_{A}$ searching an object makes a hash operation to obtain the search key (key = hash(object)). It sends a query to the $\mathrm{p} 2 \mathrm{p}$ network asking for that key. The query is routed to the peer with the most similar Peer-ID to the search key which is the one storing that key (i.e. peer $_{B}$ ). Then, peer $_{B}$ replies to peer $_{A}$, providing the IP address associated to the search key in the pair $<$ search key, peer-owner IP $>$.

Finally, peer $_{A}$ establishes a connection with the owner of the desired object and obtains it. Fig. 2 shows a p2p fully decentralized, self-organized, with semantic free index and with a ring structure.

\footnotetext{
${ }^{3}$ Key and index will be used indistinguishable during the paper.
} 


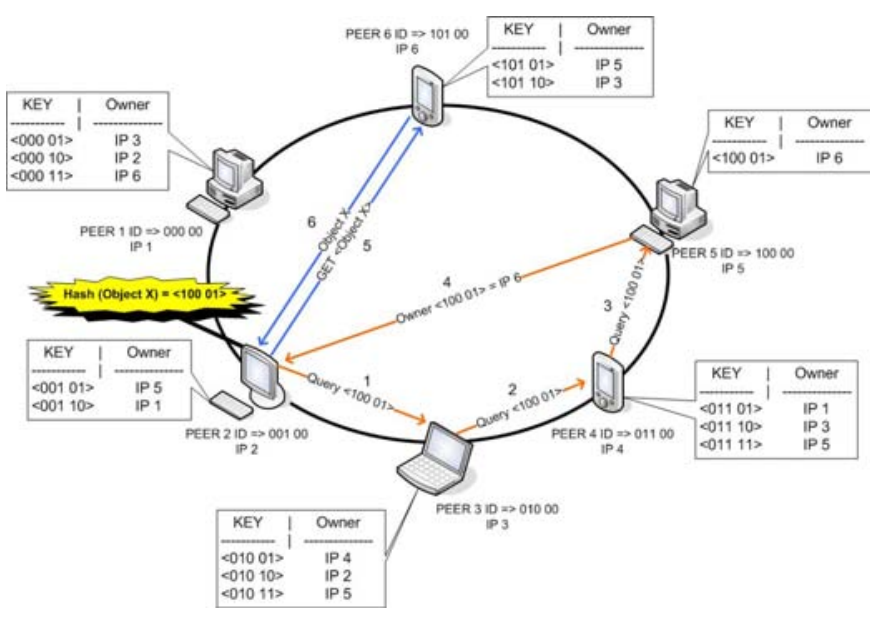

Fig. 2. P2P network scheme

\section{Proposed Solution: P2PHAN}

\section{III-A. Overview}

This section details the proposed solution: Peer-to-Peer HA Network (P2PHAN). Also, the mechanism and communication process between HAs within P2PHAN and between the HA and the Mobile Node (MN) will be analysed.

We propose the use of an overlay $\mathrm{p} 2 \mathrm{p}$ network formed by HAs in order to discover a close HA to a certain Mobile Router. Information about the location of each HA (its Autonomous System) will be available within the P2PHAN. The basic idea is that an $\mathrm{MN}$ is always associated to a HA belonging to the P2PHAN. Eventually, the MN realizes that its current HA is too far to fulfil the requirements of some kind of communication (e.g. real time ones as voice or video). Then, the MN launches the mechanism to discover a closer HA. It sends its location information (i.e. the MN's CoA) to its current HA. After that, this HA runs a search process, based on the MN's CoA, within the P2PHAN in order to discover a closer HA. The search result is the IP addresses of close HAs. Finally, the current HA informs to the MN and this one connects to one of those HAs.

The P2PHAN is composed only by HAs. At least one HA per each Autonomous System (AS) which contains foreign networks where the MRs can connect to should be part of the P2PHAN.

P2PHAN is a simple, dynamic, scalable and reliable solution. An additional important feature of the solution is the possibility to deploy it both in IPv4 and IPv6.

The paper focuses on the development of P2PHAN over NEMO, but the solution works over both Mobile IP and NEMO.

\section{III-B. P2PHAN Architecture}

P2PHAN is a self-organized, fully decentralized, with semantic free indexes and with ring structure. It is similar to Chord [10]. In our scheme the search key is hash(AS number).

When one node joins the P2PHAN, it chooses an identifier (Peer-ID) from the ids pool. Its position in the ring is determined by the chosen id, it is placed between the two nodes with the most similar id to its own id. Each peer has direct references to its two neighbours and also with other peers (crossing the ring) so as to make faster the routing within P2PHAN. Each peer uses these references to create its P2PHAN routing table.

On the other hand, each peer must store its AS number within P2PHAN. The peer obtains a key by computing the hash(AS number). Then, it looks for the peer with the most similar Peer-ID to that key and sends to this peer the key and its IP address. The destination peer stores the pair $<$ key, IP address $>$.

\section{III-C. HA Discovery}

The HA discovery mechanism could be MR initiated or HA initiated.

III-C.1. MR initiated approach: An MR connected to a $H A_{1}$ detects that the delay to this HA is higher than desired (e.g. by measuring a Round Trip Time -RTT- to $H A_{1}$ higher than a given threshold). Then, it launches the procedure to discover a closer HA. The MR sends its current CoA to $H A_{1}$. At this point, $H A_{1}$ discovers (using BGP) the CoA's AS number. Afterwards, it computes hash(AS number) which is the search key.

The search method within the P2PHAN is as follows. $H A_{1}$ sends a query with the search key. It compares the search key with the Peer-IDs stored in its P2PHAN routing table and chooses the IP address of the closest Peer-ID to the search key as next hop. The next hop operates similarly. By doing so, the last hop will be the destination peer for the query (it looks for a closer Peer-ID to the search key in its P2PHAN routing table but there is not any other node with a closer Peer-ID. This peer (e.g. $\mathrm{HA}_{2}$ ) stores the IP addresses of all the HAs located in the AS where the MR is currently attached to. Then, $H A_{2}$ sends the IP addresses of the HAs to $H A_{1}$, which sends them to the MR. The MR decides the preferred HA based on any criterion (e.g. minimum measured RTT). Fig. 3 shows the P2PHAN functionality explained above.

In order to make the P2PHAN dynamic and adaptive, every HA periodically checks if the peers which it has direct references to are still reachable and running. If it is necessary, the HA reconfigures its P2PHAN routing table and establishes new references.

On the other hand, to make the solution more robust and reliable, redundancy must be added. Therefore, each peer stores the information of its two neighbours and the MRs have a list with the IP addresses of the HAs received in the last query. Both techniques permit to avoid the loss of connectivity of one MR if its HA fails. The MR would choose one of the HAs present in its list, and neighbours of the fallen HA would restructure the ring.

Finally, the peer departure procedure is quite simple. The HA which desires to leave the P2PHAN only has to inform its MRs and its neighbours about its leaving. Then, its neighbours change the references to properly close the ring and MRs connect to a new HA. 


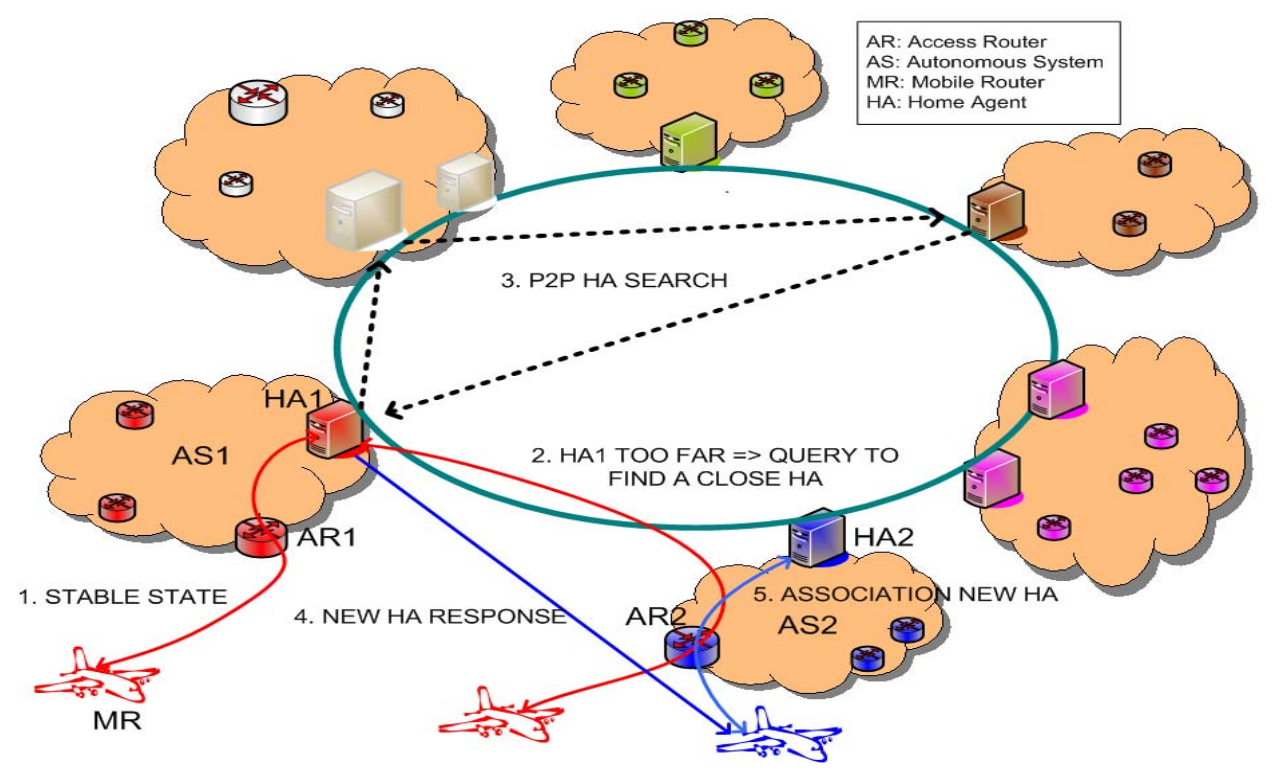

Fig. 3. P2P Home Agent Network Scheme

III-C.2. HA initiated approach: It differs from the previous case because now it is the HA who initiates the search within the P2PHAN when it receives a new CoA from the MR. After that, it sends the result (the IP addresses of the HAs in the MR's current AS) to the MR. In this case, the MR decides whether it prefers to change the HA or not.

\section{RELATED WORK}

Some solutions have been proposed for dynamic HA discovery. A global dynamic HA discovery solution, Global Dynamic Home Agent Discovery on Mobile IPv6 [11] proposes a very complex approach which needs major changes in the network infrastructure, it demands changes in devices like Border Routers or Access Routers. Besides, it proposes the use of new IPv6 anycast addresses which are not routable by routers currently deployed in the Internet.

There are also other solutions focused on local dynamic HA discovery, which involve DHCP servers [12] or Access Routers [13]. These solutions are not globally applicable. With regard to local discovery, Dynamic Home Agent Address Discovery (DHAAD) [2] also proposes a solution based on an anycast address for Home Agents in the Home Network, then the MN asks for this address in order to dynamically find a HA. These solutions are designed as local HA discovery and there are no proposals to migrate them to a global scenario.

In a nutshell, very few solutions have been proposed to solve the global dynamic HA discovery, and the proposed ones are more complex than ours. Other solutions to dynamic HA discovery were proposed for local discovery and are not useful in global discovery.

On the other side, our proposal offers a simple and global solution. It includes redundancy and techniques which make the solution more stable and robust to failures. The $\mathrm{p} 2 \mathrm{p}$ search method assures a quick answer in the HA discovery. Besides, our solution does not require infrastructure changes in devices different than HAs and MRs. Therefore, the solution can be deployed easily. In addition, our proposal works both in IPv4 and IPv6 scenarios.

\section{CONClusion AND FURTHER WORK}

Due to the sub-optimal routing introduced by the NEMO Basic Support Protocol, there are several solutions which try to solve this problem by means of finding a closer HA to the MR. This paper proposes a based $\mathrm{p} 2 \mathrm{p}$ architecture to solve the key point of these solutions that is the dynamic and global discovery of a close HA.

Several solutions have been proposed so far. Some of them are not global, and those that are global require major infrastructure changes and complex mechanisms in order to dynamically discover a closer HA.

On the other side, the solution proposed in this paper offers a dynamic and fully global mechanism to discover a close HA. P2PHAN provides a reliable and quick search method based on $\mathrm{p} 2 \mathrm{p}$ techniques. The solution is quite simple, and therefore it could be easily implemented and deployed. Another important advantage is that our proposal works both in IPv4 and IPv6.

Finally, it must be highlighted the great adaptability offered by our solution since it can be easily migrated to any service discovery scenario in a mobility environment. Basically, any scenario of mobility where the objective is dynamically discover a close server offering that service.

A future research step is to validate and evaluate by means of simulation and in a real testbed the performance of the solution, comparing it with other proposals. It must be a complete study comparing different solutions in many scenarios which could become real during next years.

On the other side, different service discovery scenarios where P2PHAN could be migrated must be analysed. It must 
be checked on each one if the proposal is feasible since some of them will have solutions which will not be improved considerably with the P2PHAN-based approach. Nevertheless, there will be others where P2PHAN migrated version will improve considerably the service discovery.

\section{ACKNOWLEDGEMENT}

The authors would like to thank Alberto García-Martínez for his insightful comments and suggestions that contributed to improve the paper.

This paper has been partially granted by the European Commission through the FP6-CONTENT-038423 Project [14] and the Spanish Government through the POSEIDON project TSI20006-12507-C03-01.

\section{REFERENCES}

[1] C. Perkins. "Mobility Support for IPv4", RFC 3344. August 2002.

[2] D. Johnson, C. Perkins, J. Arkko. "Mobility Support in IPv6", RFC 3775. June 2004

[3] V. Devarapalli, R. Wakikawa, A. Petrescu, P. Thubert. "Network Mobility (NEMO) Basic Support Protocol”, RFC 3963. January 2005.

[4] K. Leung, G. Dommety, V. Narayanan, A. Petrescu "IPv4 Network Mobility (NEMO) Basic Support Protocol", draft-ietf-nemo-v4-base-01.txt, IETF Internet Draft (work-in-progress). June 2006.

[5] Y. Rekhter, T. Li, S. Hares. "A Border Gateway Protocol 4 (BGP-4)". RFC 4271. January 2006.

[6] P. Thubert, R. Wakikawa, V. Devarapalli." Global HA to HA protocol", draft-thubert-nemo-global-haha-02.txt, IETF Internet Draft (workin-progress). October 2005.

[7] M. Bagnulo, A. García-Martínez, C.J. Bernardos, A. Azcorra."Scalable Support for Globally Moving Networks". In Proc. of 3rd International Symposium on Wireless Communication Systems (ISWCS). September 2006.

[8] E. Perera, V. Sivaraman, A. Seneviratne. "Survey on network mobility support". Mobile Computing and Communications Review, Volume 8, Number 2. April 2004.

[9] J. Risson and T. Moors: "Survey of Research towards Robust Peer-to-Peer Networks: Search Methods", Accepted to appear in Computer Networks.

[10] I. Stoica, R. Morris, D. Karger, M. F. Kaashoek, H. Balakrishnan, "Chord: A scalable peer-to-peer lookup service for internet applications," in Proc. ACM SIGCOMM'01, 2001.

[11] Y. S. Yen., C. C. Hsu, H. C. Chao."Global dynamic home agent discovery on mobile IPv6," in Wireless Communications and Mobile Computing. Volume 6, Issue 5. August 2006.

[12] H. J. Jang, A. Yegin, J. Choi. "DHCP Option for Home Agent Discovery in MIPv6", draft-jang-dhc-haopt-02.txt, IETF Internet Draft (work-inprogress). February 2006.

[13] K. Chowdhury, M. Khalil, H. Akhtar. "Home Subnet Prefix or the Home Agent discovery for Mobile IPv6", draft-chowdhury-mipv6-home-prefix00, IETF Internet Draft(work-in-progress). April 2004.

[14] "Excellence in Content Distribution Network Research. CONTENT". http://www.ist-content.org/ 